\title{
TOWARDS A CONCEPTUAL MODEL OF VALUE SYSTEMS IN COLLABORATIVE NETWORKS
}

\author{
Luis M. Camarinha-Matos \\ New University of Lishon, Quinta da Torre - 2829 Monte Caparica, PORTUGAL \\ cam@uninova.pt \\ Patrícia Macedo
}

Escola Superior de Tecnologia de Setúbal, Instituto Politécnico de Setúbal, PORTUGAL

pmacedo@est.ips.pt

\begin{abstract}
Decision making and thus the individual and joint behavior in a collaborative network depend on the underlying value system. Therefore the identification and characterization of the value system of the network and its members is fundamental when attempting to improve the collaborative process. However the concept of value system is not formally defined yet and there is not even an agreement in research community about its intuitive definition. Departing from a formal definition of value and evaluation, this paper proposes a formal conceptual model for value system and discusses its application in collaborative networks management.
\end{abstract}

\section{INTRODUCTION}

In psychology and sociology values have typically been conceptualized as shared beliefs about desired behaviors and end-states (Rokeach,1973). Value has also been defined as the "relative worth, utility, or importance: degree of excellence". This definition highlights the fact that an object's value depends on the referential that is used in its evaluation. Inside an organization, cultural and socialized values are used as referential for evaluations. Therefore, the choices and processes of an organization are directly influenced by its values. The set of values hold by an individual or society define its value system. The decision-making process in a collaborative network is naturally influenced both by the common value system of the network and the individual value system of each partner. Therefore the identification and characterization of these value systems is an important issue when attempting to improve collaborative processes. As partners have different value systems, they might have different perceptions of outcomes, which might lead to non-collaborative behavior and inter-organizational conflicts. Consequently, the development of a common value system is a significant element for the sustainability of collaboration. The definition of a value system is also an important aspect in the collaborative network operation, because it will allow the identification of the main elements that generate value in the network, and the diverse perspectives to evaluate them (Alle, 2000).

Value systems have been studied for a long time and diverse applications of the concept have been developed in several scientific areas, such as: education (Cooley, 1977), organizational management (Krishnan, 2005), and information systems design (Goguen, 2003, Shneiderman, 1998). Various authors have also referenced the value system topic in their collaborative networks studies, (Katzy, 1998, Liu, 2005, Tan, 2004), (Afsarmanesh, 2005, Filipe, 2003, Gordijn, 2000, Jamieson, 1986,

Camarinha-Matos, L., Maccdo, P., 2007, in IFIP International Fcderation for Information Processing, Volume 243, Establishing the Foundation of Collaborative Networks; eds. Camarinha-Matos, L., Afsarmanesh, H., Novais, P., Analide, C.; (Boston: Springer), pp. 53-64. 
Rezgui, 2004). However, from the analysis of these works, it can be concluded that there is no consensus about what a value system is, what elements belong to it, and how they are characterized.

The involvement of different types of stakeholders in a collaborative network, representing different interests and concerns of organizations, raises the risk of misunderstandings. It is therefore important to formalize the value system concept in order to promote a shared understanding. Moreover, formal conceptual models allow for a sounder analysis and provide a basis for the design of inter-organizational information systems that better support collaborative networked organizations (CNO). Therefore, this paper intends to give a contribution to a better understanding of this concept.

\section{VALUE SYSTEM CONCEPTUAL MODEL}

\subsection{Value and evaluation base concepts}

First a set of concepts about value and evaluation are introduced in order to allow a better understanding of the value system formal definition.

In literature the term value is used in diverse ways and for distinct purposes, often with an unclear meaning. Value in its plural form usually refers to beliefs as "an enduring belief that a specific mode of conduct or end-state of existence is personally or socially preferable to an opposite or converse mode of conduct or end-state of existence" (Rokeach,1973). Theories about values as a shared belief have been developed in Sociology and Psychology. Value in its singular form can also be used with different meanings. For instance, the concept of value involved in the mechanism of exchange is defined as: "how much is given in exchange of a product or service". This concept was first developed in the economic theories, where all products and services have an associated price that is the reference value used for the exchange. In sociological studies, Piaget (Piaget,1965) and Homans (Homans,1958) developed theories about the dynamics of value exchange, where the notion of value is extended from the specific association value-price and value-cost to a wider notion, under which, the term value is associated to "anything that can give rise to an exchange"(Piaget,1965). Also in this sense, values are not only material objects, but may also be actions, ideas, emotions, social habits, etc. For Piaget, the economic value is a quantitative value while social interactions comprise essentially qualitative values exchange. The concept of value as the utility of a product or service, considers that value comes from the qualitative characteristics of a product or service. Under this perspective, the theory of Value Analysis has developed methods for defining the value of a product, depending on its internal qualities. An object has value to the degree it fulfils its concept (Mefford,1997). Finally, the concept of value as meaning considers that something can have value not depending on its utility but depending on its significance. The meaning of a product, service or attitude depends a lot from the social and cultural context.

In an attempt to cope with the various perspectives mentioned above, the following general definition of value is proposed.

Value is the relative worth, utility or importance of something. 
In order to explain this definition of value and how it embraces various meanings of value, the concepts of evaluation object, evaluator, and evaluation have to be introduced. An evaluation object can be anything that is valuable for the evaluator. (e.g. resources, processes, behaviors, relationships, beliefs, information etc.) The evaluator is the entity that performs the evaluation. This entity can be an individual person, a social group (organization, government, virtual organization), or an instrument. Evaluation is the act of judging, measuring or calculating the quality, importance, or amount of something.

Judgment, measurement and calculation are made essentially through two basic forms: in an objective way, by applying rules and formulas to the data that characterize the evaluation object, and in a subjective way, by using mental perception about the importance, the quality or the quantity of something. In other words, the value of something depends on the function used to evaluate it. This function can be:

- A numeric function that assigns a number to an evaluation object. This number represents the value of this object in one dimension and the function implements the calculation formula of an indicator, an estimation method, or a measurement function of an instrument. The measurement process involves estimating the ratio between the magnitude of a quantity and the magnitude of a unit of the same type (e.g. length, time, mass, etc.). A measurement is the result of such process expressed as the product of a real number and a unit, where the real number is the estimated ratio. In order to define a function that implements an act of measurement, properties like monotonic, replicability and finite additivity should be studied.

- A qualitative function that represents a mental process or a qualitative judgment. This function assigns a qualitative value to something, as illustrated in Fig. 1. The properties of monotonic and replicability should also be satisfied by qualitative functions.

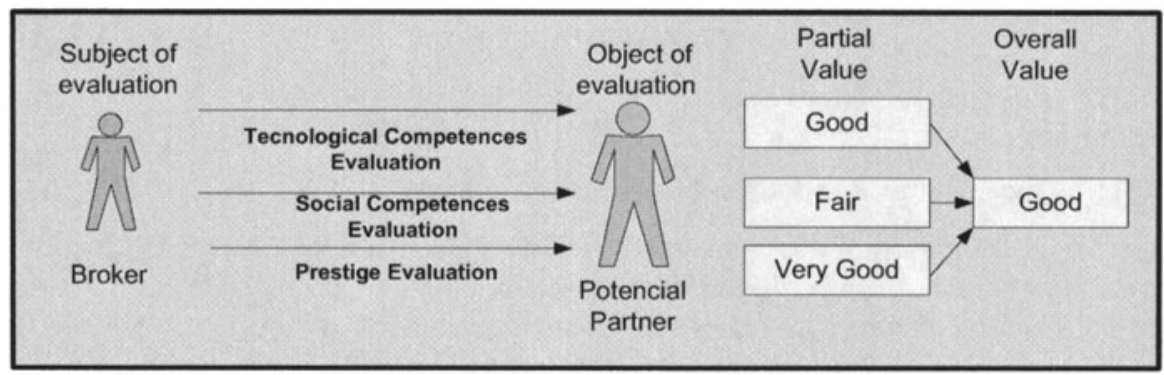

Figure 1 - Example of qualitative judgment

When making an evaluation, we are often evaluating not the overall object but a specific characteristic of this object. Therefore, it is relevant to provide a way to specify the evaluation of a particular property of an object. For instance, products, services and behaviors have several characteristics and each characteristic can be evaluated independently. When making a global evaluation, we are evaluating several characteristics and aggregating the corresponding individual values in order to reach a global value.

To better clarify this notion, two other terms are introduced: evaluation dimension and evaluation perspective. 


\section{Evaluation dimension - Characteristic of an object that is evaluated.}

The different characteristics of an object may have different degrees of importance to the actor that performs the evaluation (Note: Sociologists usually use the term "priority" to denominate the concept of degree of importance of a dimension). This idea is represented by associating, to each evaluation dimension, a weight that represents its degree of importance.

Degree of importance - Level of importance of an evaluation dimension for a given evaluator.

Depending on the objective of the evaluation, a different set of evaluation dimensions can be considered to evaluate an object, as illustrated in Figure 2. The set of selected evaluation dimensions to evaluate an object are denominated as evaluation perspective. Examples of possible perspectives:

- The business perspective, where a set of characteristics related to the business is considered.

- The social perspective, where a set of characteristics related to social, moral and cultural aspects is considered.

- The cooperation perspective, where a set of characteristics related to cooperation relationships, such as: adaptability, affinity, reliability, and agility, are considered.

The evaluation dimensions that compose a perspective can have different degrees of importance.

Evaluation perspective - A selected set of evaluation dimensions and the corresponding weights chosen to evaluate an object from a given point of view.

The following propositions are an attempt to formalize the above ideas using algebraic expressions.

(E1) Value of $\boldsymbol{x}$ is defined as the result of evaluating $\boldsymbol{x}$ with the function $\boldsymbol{f}$ $y=f(x): x \in S \wedge f \in F$ where:

(E2) $\mathrm{S}$ is the set of things that can be evaluated. if $\boldsymbol{x}$ belongs to $\boldsymbol{S}$ then $\boldsymbol{x}$ is an evaluation object.

(E3) $F$ is the set of evaluation functions and is defined as $\forall f \in F, \exists x \in S_{f}: y=f(x)$, where $S_{f}$ is composed of the elements of $S$ that can be evaluated using the function $f$. Evaluation functions can be divided into: Numeric functions and Qualitative functions. Essentially what distinguishes these two is their co-domain. $F=Q F U N F$ where:

- NF is the set of numeric functions. If $f \in N F, f$ is a numeric function defined as: $f: S_{f} \rightarrow \Re$ where $S_{f} \subset S$ The value resulted from the evaluation of $\mathrm{x}$ using the numeric function $f$ is a real number. In some cases the result of the evaluation is expressed as the product of a real number and a unit.

$$
y[u]=f(x): f \in N F \wedge y \in \Re \wedge x \in S_{f}
$$

where $u$ is the unit of measurement (e.g. $3\left[\mathrm{~ms}^{-2}\right], 10[\mathrm{~kg}]$ ).

- QF is the set of qualitative functions. If $\mathrm{f}$ belongs to QF then $f$ is a Qualitative function defined as: $f: S_{f} \rightarrow Y$

where $S_{f} \subset S \quad$ and $\quad Y=\{y 1, y 2 . . y n\}: \quad \mathrm{y}_{\mathrm{i}} \quad$ is a qualitative ordinal. 
The value resulted from the evaluation of $x$ using the qualitative function $f$ is a qualitative value.

(E4) The set of evaluation dimensions can be defined as $D=\left\{d_{1}, d_{2}, \ldots . d_{n}\right\}$ where for each dimension it is possible to define an evaluation function that evaluates it. The operator $\Phi$ is introduced, in order to express the statement: the function fallows the evaluation of the characteristic $d: f_{i} \Phi d_{i}$ where $d_{i} \in D \wedge f_{i} \in F$

(E5) An evaluation perspective can be defined as $p e=\langle d v, w v\rangle$ where:

- $d v$ is the dimensions-vector of $x$ that is defined as: $d v_{x}=\left[d_{1}, d_{2}, \ldots . d_{n}\right]: d_{i} \in D$.

This vector expresses the set of characteristics of an object that are evaluated.

- $w v$ is the weights -vector and is represented as:

$w v=\left[w_{1}, \ldots . w_{n}\right]: w_{i} \in[0 . .1] \wedge \sum_{i=0}^{n} w_{i}=1$ and $w v[i]$ is the degree of importance of $d v_{x}[i]$

Each element of the vector represents the degree of importance of the corresponding characteristic specified in $d v$. Note: The vector should be normalized in order to allow comparing the importance of a particular characteristic by distinct evaluators. For each element of the dimensions vector, a function has to be defined/ selected in order to evaluate the corresponding characteristic of the object. So, for each dimensions-vector an evaluation-vector can be specified as:

$$
f v_{d}=\left[f_{1}, f_{2}, \ldots . f_{n}\right]: f_{i} \in F \text { where } i \in[1 . . n] \wedge f v_{d}[i] \Phi d v_{x}[i]
$$

In order to represent the fact that an object can be evaluated through different perspectives, the operator $\Xi$ is defined as:

$x \Xi p e$, meaning $\boldsymbol{x}$ is evaluated through the perspective pe, where $x \in S \wedge p e \in P$ and $\mathrm{P}$ is the set of perspectives.

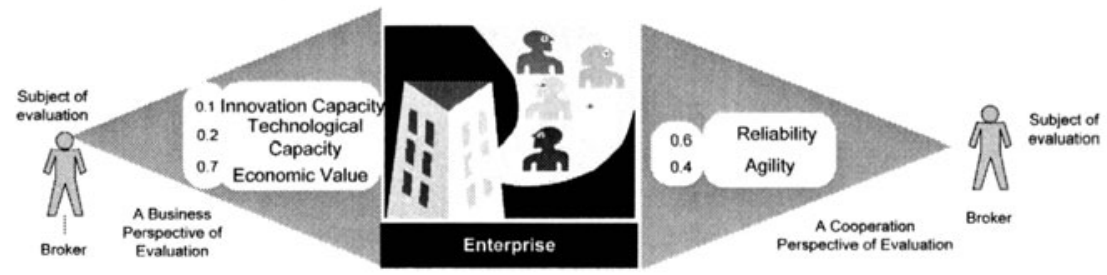

Figure 2 - Different evaluation perspectives

\section{Application example: Different evaluation perspectives}

This example does not intend to specify which dimensions should be used to evaluate a partner. It simply tries to illustrate the application of the evaluation perspective concept as it was defined in (E5).

Suppose that a CNO manager wants to evaluate a partner (an enterprise). Distinct evaluation perspectives can be defined. In the case illustrated in Figure 2 a Business Perspective and a Cooperation Perspective are shown. Depending on the purpose of the evaluation the manager selects the evaluation perspective.

$d v_{l}=[$ Innovation Capacity, Technological Capacity, Economic Value $]$ $w v_{1}=[0.1,0.2,0.7]$

Evaluation Business Perspective $=\boldsymbol{p e}_{\text {business }}\left\langle d v_{l}, \mathrm{w}^{\prime} v_{l}\right\rangle$

$d v_{2}=$ [Reliability, Agility]

$w v_{2}-[0.6,04]$

Evaluation Cooperative Perspective $=p e_{\text {cooperation }}-\left\langle d v_{2}, w v_{2}\right\rangle$ 


\subsection{A definition of value system}

Before defining the concept of value system it is necessary to introduce some auxiliary definitions:

A system is an assemblage ${ }^{1}$ of inter-related elements comprising a unified whole. Any element which has no relationship with any other element of the system cannot be a part of that system. So a system can be formalized as a duple:

(E6) $S Y S=\angle E L, R E>$ where $E L$ is set of elements and $R E$ is the set of relationships among the elements of $E L$.

(E7) Value objects subsystem (OS) is a system composed of the objects that can be evaluated and can be defined as a duple: $O S=\langle S, R S\rangle$ where:

- $S$ is the set a valuable things

- $R S$ is the set of relationships among the elements of $S$, which can be essentially of two types: composition and specialization.

(E8) Evaluation subsystem (ES) is a system composed of all elements that represent "mechanisms" of evaluation (functions, dimensions and perspectives) and can be defined as a duple:

$E S=<E F, R E>$ where EF represents all the elements that belong to the evaluation subsystem and is defined as a triple: $E F=\langle F, D, P>$ where:

- $F$ is the set of evaluation functions; $D$ is the set of evaluation dimensions and $P$ is the set of evaluation perspectives.

- $R E$ is the set of relationships among the elements of $E F$. These relationships can be categorized as:

oComposition-relation - One function is defined by aggregation of two or more functions.

○ Evaluates-relation - The relation is specified by the operator $\Phi$, that specifies that a function can be used to evaluate a specific dimension.

$\circ$ Priority-relation - The relation that specifies the degree of importance of a characteristic in an evaluation perspective.

Using these auxiliary concepts, we can now formally define a value system.

(E9) A value system (VS) is composed of an aggregation of two subsystems, the value objects subsystem and the evaluation subsystem, and the set of relations established between them. Thus VS can be specified as a duple $V S=\langle E V S, R V S\rangle$ where:

- $E V S=<O S, E S>$ is the aggregation of the two subsystems that compose the value system. $O S$ is the set of valuable things for an organisation and $E S$ represents the set of mechanisms used for their evaluation under different perspectives, where each perspective is composed of a weighted set of dimensions of evaluation.

- $R V S$ represents the set of relationships between the two sub-systems. According to the systems theory, if there is a relation between two elements of different subsystems, this implies that these two subsystems are related. These two subsystems are related by two categories of relationships:

$\circ$ Value-relation - What relates a function and an object is the value resulting from evaluating the object using that function.

o Perspective-relation - The relation that is defined by the operator $\Xi$, that specifies that an object is evaluated through a given evaluation perspective.

\footnotetext{
${ }^{1}$ assemblage -a collection of things or a group of people or animals, in Cambridge Dictionary.
} 
This definition of value system can be applied to a CNO. Considering that a $\mathrm{CNO}$ is composed of several organisations, it is possible to define the value system for each organization and the value system for the network as a whole.

The value system of a $\mathrm{CNO}$ is defined as:

(E10) $V S_{\text {CNO }}=<E V S_{C N O}, R V S_{C N O}>$ where $E V S_{\mathrm{CNO}}$ is composed of the two subsystems:

- Value objects subsystem $\left(O S_{C N O}\right)$, containing the set of CNO valuable things such as financial resources, human resources, processes, information, relationships with external entities, brands.

- Evaluation subsystem $\left(E S_{C N O}\right)$, containing the set of evaluation functions $\left(F_{C N O}\right)$; the set of characteristics that are going to be evaluated for each object $\left(D_{C N O}\right)$, and the set of evaluation perspectives used in the CNO $\left(P_{C N O}\right)$.

Each CNO should define the order and prioritization of the dimensions of evaluation that will be used during its life-cycle. The set of functions, dimensions and perspectives of evaluation should be defined during the CNO set-up and all partners should know and agree on them. The change of one of these elements during the $\mathrm{CNO}$ operation should be done with the agreement of all partners.

For some CNO processes not just the specification of the CNO value system is relevant, but also the specification of the value system of each partner can be useful. For example, in the partner's selection process, in order to choose the partner that best fits a coalition in terms of shared common beliefs, it is necessary to know both the value system of the candidate members and of the planned CNO.

Application example: Definition of order and prioritization of core-values in a CNO

This example does not intent to specify which "core values" should belong to the Value System of any CNO. It simply illustrates how the set of "core values" can be specified according to the proposed modeling approach. The shown "core values" are based on the findings of a survey concerning the definition of value systems performed by ECOLEAD (Romero, 2006).

The definition of the set of evaluation dimensions that are important to the members of the $\mathrm{CNO}$ and that guide all actions should be specified during its configuration. This notion of order and prioritization of "core values" can be mapped onto our conceptual model by the evaluation perspective. Imagine that a CNO defines that the most relevant attributes for the network are: Quality, Reliability, Flexibility, Innovation (see Figure 3). These four attributes do not have the same degree of importance. Thus the CNO should specify the degree of importance of each attribute and all members of the $\mathrm{CNO}$ should be aware that all decisions and behaviors should be in accordance to this perspective of evaluation.

For each dimension of evaluation (each attribute) an evaluation function should be defined in a collaborative way. The definition of methods to evaluate agility, quality, reliability and flexibility are not standard, and several studies proceed in theses areas.

In the proposed conceptual model the specification of the "core values" can be done as represented above. The value system $\left(V S_{C N O I}\right)$ is composed of an aggregation of two subsystems the value objects subsystem $\left(O S_{C N O I}\right)$ and the evaluation subsystem $\left(E S_{C N O I}\right)$, and the set of relations established between them $\left(R V S_{C N O I}\right)$.

$V S_{C^{\prime O} 1}=<E V S_{C N O 1}, R V S_{C N O 1}>$ where $E V S_{C N O 1}=<O S_{C N O 1}, E S_{C N O 1}>$.

- All resources, activities, beliefs and behaviour that have value to the $\mathrm{CNO}_{1}$ are elements of the subsystem $O S_{\mathrm{CNOI}}$.

- $\{$ Quality, Innovation, Flexibility, Reliability $\} \subset \mathrm{D}_{\mathrm{CNO}}$ - Evaluation dimensions of the $\mathrm{CNO}_{1}$.

- $p e_{\text {core }}=\langle d v, w v\rangle \in P_{C N O I}$ All the evaluation functions $\left(F_{C N O I}\right)$, evaluation dimensions 
$\left(D_{\mathrm{CNOI}}\right)$, evaluation perspectives $\left(P_{\mathrm{CNO}}\right)$, and the set of relations established among the elements of these three sets define $E S_{C N O I}$.

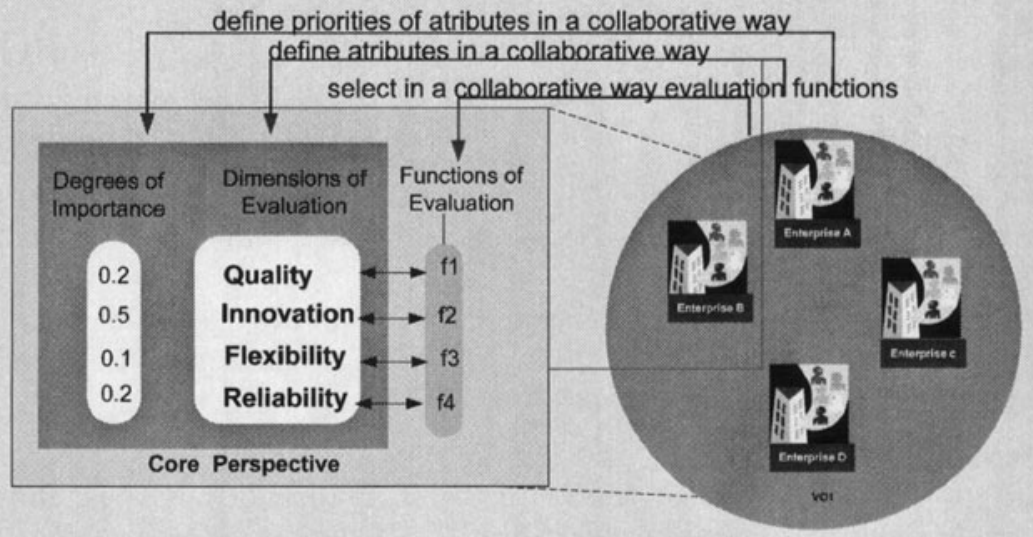

Figure 3 - Core-values definition in a $\mathrm{CNO}$

- The perspective $p e_{\text {core }}$ is used to evaluate the network where $d v=[$ Quality , Innovation, Flexibility, Reliability] and (e.g.) $w v=[0.2,0.5,0.2,0.1]$.

- $\left\{f_{1}, f_{2}, f_{3}, f_{4}\right\} \subset F_{C N O I}$ - These evaluation functions belong to the elements of the subsystem $E S_{\text {CNOI }} . \quad f v=\left[f_{1}, f_{2}, f_{3}, f_{4}\right]$ is a functions' vector that contains the evaluation functions selected to evaluate the four evaluation dimensions defined in $d v$. Just as an example, the following functions are considered:

$f_{1}: S_{x 1} \rightarrow \Re_{0} f_{1}=$ percentage of services and materials deliverable without any complain.

$f_{2}: S_{x 1} \rightarrow \Re_{0} f_{2}=$ number of industrial patents

$f_{3}: S_{x 1} \rightarrow \Re_{0} f_{3}=$ average time to develop or change a logistic process

$f_{4}: S_{x 1} \rightarrow \Re_{0} f_{4}=$ percentage of time without process faults.

$\mathrm{RVS}_{\mathrm{CNO} 1}$ is composed of the relations among the two subsystems. The relation that specifies that the $\mathrm{CNO}_{1}$ is evaluated through the evaluation perspective pe core belongs to RVS $_{\mathrm{CNOl}}$.

\subsection{Related work}

One of the aims of the proposed conceptual model is to offer a framework that embraces, in a coherent way, diverse assumptions about value and value system. In this subsection the proposed conceptual model is compared to related work.

Goguen and Linde have developed, since 1978, several studies about value and value system in organizations (Goguen,1994) (Goguen, 2003). They introduced a method for using discourse analysis to determine the value system of an organization from a collection of stories told by members of the organization among themselves on informal occasions. The evaluative material collected from the stories is classified and represented using a formal structure called a value system tree. A value system tree (Goguen, 1994) serves as a formal summary of the interpretation that the analysts gave to data that has been collected. It is possible with the conceptual model proposed above to represent a value system tree, but the formal model proposed by Goguen has several limitations: it only considers one type of relationship between values (hierarchical relationship); it does not make a distinction 
between the value and the characteristic that is evaluated; it does not represent the evaluation function.

Romero et al. (Romero, 2006) have proposed a multi-value system model for VO Breeding Environments as part of the business model. The multi-value system includes the definition of different values: economic, social, and knowledge; and the identification of the stakeholders' participation in the value generation process. The work presented by these researchers does not include a formal specification of value system, neither a clear definition of value. They classify values in: financial, social and knowledge, but some times they also use the term value with the meaning of valuable object. This model for value system does not include the concept of different degrees of importance and is focused essentially on the identification of value transactions.

Another contribution to the study of value systems comes from the Distributed Artificial Intelligence discipline, which has developed some theories about value systems using agents (Filipe, 2003), (Antunes, 2000), (Rodrigues, 2005). Filipe (Filipe, 2003) proposed an approach based on organizational agents where it is assumed that an agent it responsible for its values. The agent's preferences with respect to norms are defined in its value system. In this approach an agent can represent a member of an organization or an organization itself. The norms that define the preferences are formally represented using Deontic Logic. This approach to value systems limits the specification to the definition of preferences and does not include the notion of evaluation functions and evaluation dimensions.

The work done by (Rodrigues,2005) is based on the Piaget theories (Piaget, 1965) on values exchange and is concerned with the dynamics of values in interactions. The focus of our conceptual model is not the representation of value exchange mechanisms, but rather the definition of evaluations functions and dimensions, thus providing the base elements to specify value transaction between actors.

Gordijn, Yao-Huan Tan and Kartseva (Gordijn, 2000), (Tan, 2004), (Kartseva, 2004) have developed a methodology and an ontology called e3-value in order to define value models that support the business processes. This ontology has introduced the concepts of value object and value activity. It defines an actor as an independent entity that adds value to the system with the performance of value activities. An actor is an economic or legal entity that is engaged in business transactions and exchanges value objects. A value object is defined as a service, thing, or consumer experience that is of value to one or more actors. Each value object has one or more valuation properties that are characterized by a name and a unit that indicates the scale of evaluation. The e3-value model was developed to support e-commerce business and is essentially focused on the economic value of objects and on activities and actors that create economic value (Gordijn, 2000). One important issue in collaboration sustainability is the agreement about the shared values (Afsarmanesh,2005), but as the e3-value model does not support the idea of value system as the ordering and prioritization of values, it is not possible with such model to analyze if the members of a CNO share the same values. Another relevant point in CNO management is the agreement among CNO members about the method used to evaluate each object property. This aspect cannot be supported directly with the e3-value model, because it does not consider the specification of evaluations functions, neither the specification of the relationships between functions and characteristics to be evaluated. 


\section{FUTURE WORK AND RESEARCH ISSUES}

The proposed conceptual model of a value system for CNOs includes a set of complex entities; some of them may require the adoption of soft modeling perspectives and techniques in order to allow a proper understanding and management. One interesting possibility is to develop a hybrid system, where some entities of the model are represented using crisp techniques and others using soft computing ones. Soft computing methods can exploit the tolerance for imprecision, uncertainty and partial truth (Berthold,2003). Therefore such methods are promising candidates to deal with aspects related to qualitative evaluation and priority of values. For these two aspects fuzzy logic, which derived from the fuzzy sets theory, seems to be a promising approach. Its objective is to allow degrees of inclusion/relevance of each element in a given set. It means to allow one element to belong in a given set in a bigger or smaller intensity, also known as degree of membership or degree of truth. For instance, to calculate the overall value of an object using a specific evaluation perspective, we can use fuzzy rules to combine the partial values and their weights of importance to infer an overall value

When partners have different value systems, which typically leads to different perceptions of values, non-collaborative behaviors are likely to develop. Consequently, the development of a common value system in a CNO context is an important step to support the sustainability of a collaborative behavior over time (Abreu, 2006). In order to overcome this problem, mechanisms to reason about the compatibility among value systems should be developed. The first step is to specify forms of comparison or identification of relationships between value systems of different members. Causal modeling seems to be a good tool to deal with this. This method has naturally grown due to a need for a sketching technique to support and facilitate reasoning about cause and effect. It builds upon a binary relationship, called an influence relationship, between two entities that represent named quantitative or qualitative value or value set. Whereby changes in the influencing entity are conveyed as changes in the influenced entity (Greenland, 2002).

Example: Analysis of influence between values

The causal modelling method may be used to model:

- Causal relations among organizational values- this will allow to analyze the influence among values. For example it is possible to specify, for a university, that the value of Knowledge influences positively the value of Prestige.

- Inter-relations of influence between values of distinct members. An university and an industry are two types of organization that are expected to give different priorities to their evaluation dimensions. In spite of this, it is possible that a sustainable collaborative relationship is established between them. So different influence relationships between values systems do not mean that their level of alignment is low.

In the example illustrated in Fig. 4 three inter-relations of influence are represented:

- Industry-prestige influences positively the University-prestige. If a University collaborates with a prestigious industry then the value of prestige of the university tends to increase.

- University-prestige influences positively the Industry-prestige. If an industry collaborates with a prestigious university then the level of prestige of the industry tends to increase.

- University-Knowledge influences positively the Industry-Technological Capacity. If an industry collaborates with a university with a high degree of knowledge then the university tends to "transfer" its knowledge to industry in form of best-practices, developing the technological skills of industry worker and consequently the technological 
capacity level will increase.

The degree of importance of each value is also relevant and should be represented. In the example represented in Fig. 4, the industry does not consider the technological capacity as a priority. So, in spite of the positive causal inter-relationship between University-Knowledge and Industry-Technological Capacity, this inter-relationship does not contribute to the alignment of the two value systems, because Industry will not value the knowledge contribution from the university.

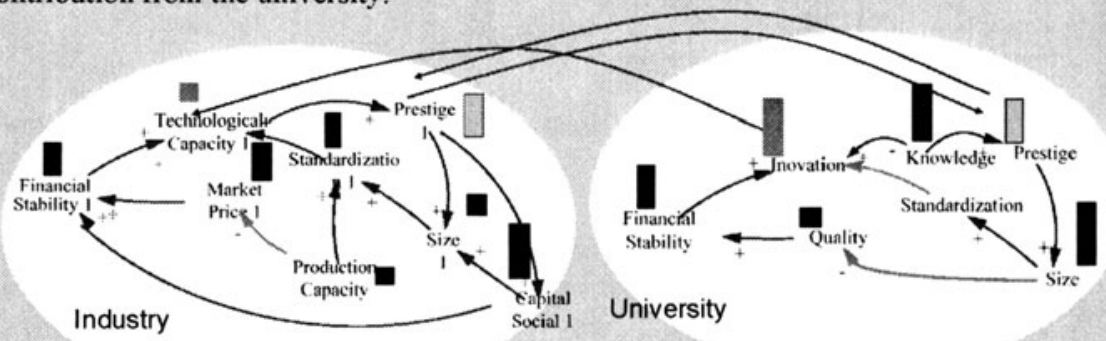

Figure 4 - Example of causal models to represent the influences among values

The processes of analyzing and formulating policies for CNOs are an important and critical issue, which must consider the influence and causal effect, which may lead to their effectiveness or ineffectiveness. In the process of formulating policies, all important values of all stakeholders must be considered. The causal analysis can be applied in order to understand the causal effects in each partner of applying some policies depending on the value system of each partner.

Another research issue that is very important is the development of a methodology to define a value system for a CNO in a collaborative way. As mentioned above, one of the difficulties about value system definition is the ambiguity about the meaning of values and evaluation characteristics so, this methodology has to be supported by collaborative negotiation and meaning negotiation. The work done in the areas of meaning negotiation (Moor, 2006), meaning evolution, and ontology's alignment (Kotis, 2004) may give some hints in order to define a proper methodology for common value system definition in CNOs.

\section{CONCLUSIONS}

The characterization and understanding of value systems is a key condition for improving the sustainability of collaborative networks. In this context and considering the notions of value system suggested in different disciplines, an attempt to provide a comprehensive formal conceptual framework was made. The conceptualization of value system is expected to promote a shared understanding among the different stakeholders of the network. This framework is also suggested as the baseline for further research and development in the area, namely in what concerns the issue of alignment or compatibility of value systems.

Acknowledgments - This work as supported in part by the ECOLEAD integrated project funded by the European Commission. 


\section{REFERENCES}

1. Abreu A, Camarinha-Matos LM. On the Role of Value Systems and Reciprocity in Collaborative Environments. In: Spring, ed. IFIP, Volume 224, Network-Centric Collaboration and Supporting Frameworks: Boston Springer, 2006.

2. Afsarmanesh H, Camarinha-Matos LM. A framework for Management of Virtual Organization Breeding environments. In: Collaborative Networks and their Breeding Environments: Springer, 2005.

3. Alle V. Reconfiguring the Value Network. Journal of Business Strategy 2000;21.

4. Antunes L, Coelho H, Faria J. Improving choice mechanisms within the bvg architecture. In: Lesprance CCaY, ed. Agent Theories, Architectures, and Languages - ICMAS 2000; 2000: Springer Verlag, 2000: 209-304.

5. Berthold MR. Intelligent Data Analysis: An Introduction: Springer, 2003.

6. Cooley CR. Cultural Effects in Indian Education: An Application of Social Learning Theory. Journal of American Indian Education 1977;17.

7. Filipe J. The organisational semeiotics normativa paradigma In: Collaborative Networked Organizations. London: Springer, 2003: 261-272.

8. Goguen J. Requirements Engineering as the Reconciliation of Technical and Social Issues. In: Requirements engineering: social and technical issues, 1994: 162-199.

9. Goguen J. Semiotics, compassion and value-centered design. In: Keynote lecture, in Proceedings of the Organizational Semiotics Workshop, University of Reading; 2003; UK, 2003.

10. Gordijn J, J.M. Akkermans, Vliet JCv. Value based requirements creation for electronic commerce applications. In: In Proceedings of the 33rd Hawaii International Conference On System Sciences; $2000,2000$.

11. Greenland S, Brumback B. An overview of relations among causal modeling methods. international journal of epidemiology 2002.

12. Homans GC. Social Behavior as Exchange. American Journal of Sociology 1958; 63:597-606.

13. Jamieson M, A. T, . TA. Refocusing collaboration technologies in the construction Value System. In: JCIB W78 Conference Construction on the Information Superhighway, 1986; Slovenia, 1986: 279-289.

14. Kartseva V, Gordijn J, Akkermans H, . A Design Perspective on Networked Business Models: A Study of Distributed Generation in the Power Industry Sector. In: 12th European Conference on Information Systems; 2004, 2004.

15. Katzy B. Value System Redesign. ACM SIGGROUP Bulletin archive, 1998;19: 48-50.

16. Kotis K, Vouros GA. The HCONE Approach to Ontology Merging. In: The Semantic Web: Research and Applications. First European Semantic Web Symposium (ESWS 2004); 2004, 2004: 137-151.

17. Krishnan VR. Leader - Member Exchange, Transformational Leadership, and Value System. EJBO Electronic Journal of Business Ethics and Organization Studies 2005;10.

18. Liu P-Y. A study based on the Value System for the interaction of the multi-tiered supply chain under the trend of e-business. In: The 7th international conference on Electronic Commerce; 2005, 2005.

19. Mefford D, Meffortd V. Values Usage Exercise (VUE) A Tool For Raising Values Awareness ConcerningThe Professional - Personal Values Interface. In: Conference on Professional Ethics. Washington, 1997.

20. Moor AD, De Leenheer P, Meersman M. DOGMA-MESS: A Meaning Evolution Support System for Interorganizational Ontology Engineering. In: 14th International Conference on Conceptual Structures (ICCS 2006); 2006; Aalborg, Denmark: Springer-Verlag, 2006: 189-203.

21. Piaget J. Essay sur la Th'eorie des Valeurs Qualitatifs en Sociologie Statique. Paris: Librairie Droz, 1965.

22. Rezgui Y, Wilson I, Olphert W, Damodaran L. Socio-Organizational issues. In: Collaborative Networked Organizations: Springer 2004, 2004: 187-198.

23. Rodrigues MR, Luck M. Analysing Partner Selection Through Exchange Values. In: MABS; 2005: J.S. Sichman and L. Antunes, 2005: 24-40.

24. Rokeach M. The nature of human values. New York: Free Press. 1973.

25. Romero D, Galeano N, Giraldo J, et al. Ecolead WP2, D21.4 Characterization of VBE Value System and Metrics, 2006.

26. Shneiderman B. Designing the User Interface: Strategies for Effective User Interface Interaction, 3rd ed: Addison Wesley Longman, 1998.

27. Tan Y-H, Thoen W, Gordijn J. Modeling Controls for Dynamic Value Exchange in Virtual Organizations. In; 2004, 2004. 